\title{
Expanding and understanding metabolite space
}

\author{
Julio E Peironcely ${ }^{1 *}$, Andreas Bender ${ }^{2}$, M Rojas-Chertó ${ }^{2}$, T Reijmers $^{2}$, L Coulier $^{1}$, T Hankemeier $^{2}$ \\ From 5th German Conference on Cheminformatics: 23. CIC-Workshop \\ Goslar, Germany. 8-10 November 2009
}

In metabolomics the identity and role of low mass molecules called metabolites that are produced in cell metabolic processes are investigated. These make them valuable indicators of the phenotype of a biological system. The 'Metabolite Space' is the total chemical universe of metabolites present in all compartments and in all states from any organism. These molecules exhibit common features that form what can be called 'metabolite likeness'. Here, we focus on the human metabolite space, including both endogenous and exogenous (such as drug) metabolites. In order to analyze the 'Metabolite Space', we collected data from the Human Metabolome Database (HMDB) [1] which is a comprehensive database for human metabolites containing over 7000 compounds that were identified in several human biofluids and tissues. As there still remain many compounds to be identified that lay outside the boundaries of this known space, exploring this unknown region is crucial to evaluate 'metabolite likeness'.

In order to expand 'Metabolite Space' in our approach we employed the Retrosynthetic Combinatorial Analysis Procedure (RECAP) [2] to generate new molecules that possess features similar to those present in metabolites, however in other (but still likely) rearrangements. We studied how discernible these new molecules are from real metabolites and, hence, whether synthetic organic chemistry reactions are indeed able to expand the known universe of metabolites. We further studied the new chemistry present in the expanded metabolite space by looking at Murcko assemblies [3], ring systems and other chemical properties. The new metabolite space is compared to other small molecules, such as those obtained from the ZINC database, that are not metabolites. By combining all the above analyses we expect to characterize better the metabolite space, and furthermore, to predict the metabolite-likeness of a molecule and to understand its immanent properties.

${ }^{1}$ TNO, Quality of Life, Utrechtseweg 48, Zeist, The Netherlands

\section{Author details}

${ }^{1}$ TNO, Quality of Life, Utrechtseweg 48, Zeist, The Netherlands. ${ }^{2}$ Netherlands Metabolomics Centre, University of Leiden, LACDR, Einsteinweg 55, 2333 CC Leiden, The Netherlands.

Published: 4 May 2010

\section{References}

1. Wishart DS, et al: Nucleic Acids Res 2007, 35 Database: D521-526.

2. Lewell XQ, Judd DB, Watson SP, Hann MM: J Chem Inf Comput Sci 1998, 38:511-522.

3. Bemis GW, Murcko MA: J Med Chem 1996, 39:2887-2893.

doi:10.1186/1758-2946-2-S1-P39

Cite this article as: Peironcely et al:: Expanding and understanding metabolite space. Journal of Cheminformatics 2010 2(Suppl 1):P39. 